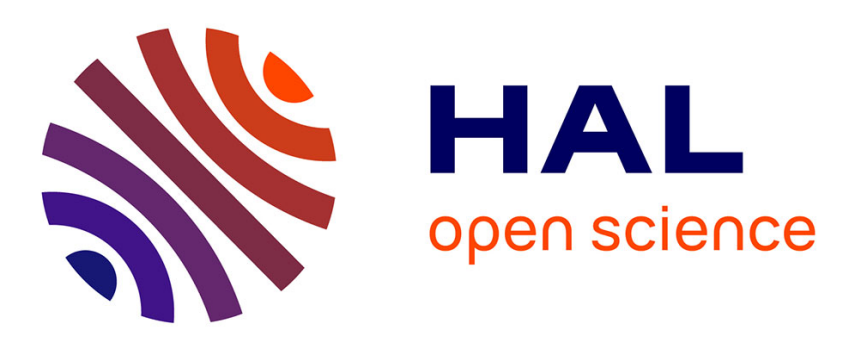

\title{
Disturbances Classification based on a Model Order Selection Method for Power Quality Monitoring
} Zakarya Oubrahim, Vincent V. Choqueuse, Yassine Amirat, Mohamed Benbouzid

\section{- To cite this version:}

Zakarya Oubrahim, Vincent V. Choqueuse, Yassine Amirat, Mohamed Benbouzid. Disturbances Classification based on a Model Order Selection Method for Power Quality Monitoring. IEEE Transactions on Industrial Electronics, 2017, 10.1109/TIE.2017.2711565 . hal-01576981

\section{HAL Id: hal-01576981 \\ https://hal.univ-brest.fr/hal-01576981}

Submitted on 24 Aug 2017

HAL is a multi-disciplinary open access archive for the deposit and dissemination of scientific research documents, whether they are published or not. The documents may come from teaching and research institutions in France or abroad, or from public or private research centers.
L'archive ouverte pluridisciplinaire HAL, est destinée au dépôt et à la diffusion de documents scientifiques de niveau recherche, publiés ou non, émanant des établissements d'enseignement et de recherche français ou étrangers, des laboratoires publics ou privés. 


\title{
Disturbances Classification based on a Model Order Selection Method for Power Quality Monitoring
}

\author{
Zakarya Oubrahim, Student Member, IEEE, Vincent Choqueuse, Member, IEEE, Yassine Amirat, Member, IEEE and \\ Mohamed Benbouzid, Senior Member, IEEE
}

\begin{abstract}
In this paper, a new technique for power quality disturbances classification is proposed. It focuses on voltage sags and swells that are first pre-classified into four classes that depend on the number of non-zero symmetrical components and can contain different types of sag and swell. Using the estimated symmetrical component values, we can afterward classify the corresponding sag or swell signature. In this study, we show that the pre-classification can be reformulated as a pure model order selection problem. To solve this problem, we propose two preclassifiers based on Information Theoretical Criteria. The former yields the highest statistical performances, while the latter has a lower computation complexity. The performances of the proposed classification algorithms are evaluated using Monte Carlo simulations on synthetic signals and using real power system data obtained from the DOE/EPRI National Database of Power System Events. The achieved simulations and experimental results clearly illustrate the effectiveness of the proposed algorithms for voltage sag and swell classification.
\end{abstract}

Index Terms-Power quality monitoring, disturbances, voltage sags and swells, estimation, classification, symmetrical components, unbalanced power system.

\section{INTRODUCTION}

$\mathbf{O}$ VER the last decades, the necessity of producing more energy combined with the interest in clean energies have led to a greater penetration of renewable energy systems in the power grid. The intermittent and discontinuous nature of renewable energy sources may impact the power system and can lead to grid instability or even its failure. Particularly, the integration of renewable energy sources has a substantial influence on power quality by affecting voltage and frequency control [1]-[4]. To overcome this difficulty, the need of a better control strategies for power systems have recently led to the concept of smart-grids [5]. A smart grid uses two-way flows of electricity and information, and computational intelligence in an integrated fashion across the entire spectrum of the energy system from the generation to the end points of electricity consumption [6]. A smart-grid is a modern electric power grid infrastructure for improved efficiency, reliability, and safety, through automated control and modern communication technologies. Furthermore, the future smart grid is expected to be self-healing and resilient to system anomalies [7]-[10].

A key role of a smart grid is allowing an electrical grid to be more flexible while meeting power quality standards. Power Quality (PQ) is a very significant issue of electrical power system operations. PQ disturbances can be extremely different in their characteristics and consequences for end-user equipments. They are usually divided into several classes such as sag, swell, transient,

Z. Oubrahim, V. Choqueuse, and M.E.H. Benbouzid are with the University of Brest, FRE CNRS 3744 IRDL, Brest, France (e-mail: zakarya.oubrahim@univ-brest.fr, vincent.choqueuse@univ-brest.fr, mohamed.benbouzid@univ-brest.fr). Z. Oubrahim is also with ISEN Brest, Brest, France. M.E.H. Benbouzid is also with the Shangha Maritime University, Shanghai, China. Y. Amirat is with ISEN Brest, FRE CNRS 3744 IRDL, Brest, France (e-mail: yassine.amirat@isenbretagne.fr). This work was supported by Brest Métropole and ISEN Brest. fluctuation, and interruption [11]. Voltage sags and swells are one of the most critical disturbances in power supply because they can degrade customer load performance and efficiency. From an economic viewpoint, these disturbances can cause million-dollar losses for industrial consumers [12].

In terms of power quality issues, this paper focuses on voltage sags and swells. There are several international standards for characterizing voltage sags and swells, such as the IEEE 1159, IEC 610004-30, and EN 50160 [13]-[15]. According to the IEEE std. 1159, a sag is a decrease in the root mean square (rms) voltage or current levels from 0.1 to 0.9 per unit $(\mathrm{pu})$ at the power system frequency for durations ranging from a half-cycle to one minute (short time), or over one minute (long time). According to the same standard, a swell is an increase in the rms voltage level between 1.1 and 1.8 pu of the nominal value. To minimize their economical impacts, it becomes imperative to integrate advanced algorithms to characterize voltage sags and swells. This characterization is usually composed of two steps which are the event detection, that is concerned with determining the starting and ending times of the event, and the event classification [11].

Several techniques have been proposed in the literature to detect three-phase unbalance and/or classify voltage sags and swells. Regarding the detection of three-phase unbalance, [16] and [17] describe two techniques based on hypothesis tests. The classification of PQ disturbances have also been investigated in [18]-[21], where twovoltage classification algorithms were proposed. These algorithms allow classifying the six voltage sag types $C_{a}, C_{b}, C_{c}, D_{a}, D_{b}$, and $D_{c}$ according to the ABC classification proposed in [22]. The first classifier is based on symmetrical components (SC) and the second classifier is based on three-phase voltage magnitudes and their phase angles (TP-TAA). The classifier described in [19] and T18 compares the six rms values of phase-to-ground and phase-tophase voltages after removing the zero-sequence component. This six-phase algorithm also classifies the voltage sag types $C_{a}, C_{b}, C_{c}$, $D_{a}, D_{b}$, and $D_{c}$. Despite their simplicity, these techniques are very sensitive to large variations in magnitude or phase-angle jump and they do not cover all sag types proposed in [22]. In [23], the authors have proposed a technique based on space vector representation in the complex plane and zero sequence voltage. This technique provides a complete sag and swell type classification. A fault detection and classification technique using wavelet transform (WT) has been also proposed in [24].

A panorama and state of the art of PQ disturbances classification based on pattern recognition techniques has been proposed in [29]. Classifiers based on pattern recognition techniques, such as artificial neural networks (ANN) or support vector machine (SVM), have been presented in [25]-[28]. In [30], a PQ disturbances classifier based on balanced neural tree method and using empirical-mode decomposition (EMD) has been presented. However, the EMD suffers from drawbacks such as mode mixing that may lead to a wrong intrinsic mode functions (IMF) decomposition, and border effects. In [31], the authors have proposed a PQ disturbances analysis method based on wavelets and fuzzy sets theory. Nevertheless, the 
wavelet transform technique leads to poor performances accuracy under noisy environment. In [32], a hybrid fuzzy C-means particle swarm optimization (PSO) technique has been proposed for PQ disturbances classification using a variety of windowing techniques. This technique uses the S-transform, which is an extension of the wavelet transform (WT). An approach for PQ disturbances detection and classification under non-stationary conditions based on S-transform and neural networks has also been presented in [33]. In [34], the authors have developed an S-transform-based probabilistic neural network $(\mathrm{PNN})$ classifier for recognition of PQ disturbances. A combination of a neural-network-based technique for single and combined PQ disturbances detection and classification under noiseless and noisy conditions has been proposed in [35]. Although this classifier has good capabilities for classifying combined PQ disturbances, the analysis stage needs more attention about subclasses of each PQ disturbance, especially sag and swell. A feature extraction method for the automatic classification of PQ disturbances based on wavelet neural network (WNN) has been proposed in [36]. Its main advantage is the reduction of training data size and a good signal characterization. A SVM classification system for voltage disturbances has been proposed in [37], where the SVM classifier performances is investigated for different training and testing data. However, the performances of all the above-presented artificial intelligence techniques critically depend on the learning stage that requires a training database. The size of this latter must be sufficiently large to encompass different kinds of power quality conditions, which may be difficult to obtain in practice. Their performances also depend on many parameters and on the features extraction process. Furthermore, the learning stage requires relatively high computational complexity effort. Moreover, most of previous works in PQ disturbance classification are suboptimal because they only exploit the information contained in a single-phase, instead of exploiting the multidimensional nature of the electrical signals [38].

To overcome these problems, this paper proposes a new PQ classification technique based on the analysis of the three-phase signal. The proposed classifier can be applied as a post-processing tool for transient characterization and disturbance detection in power systems [11]. Similarly to the technique described in [17], the proposed classifier is based on model order selection. Nevertheless, unlike the method in [17], which mainly focuses on the detection problem, the proposed technique emphasizes on the classification problem. Specifically, the proposed classifier is able to identify the number of non-zero symmetrical components and to pre-classify the signal into 4 classes under quasi-stationary conditions. Once the correct class is selected, the corresponding sag or swell can be identified using the estimated symmetrical components. The performances of the proposed algorithm are evaluated with synthetic and experimental data. Thus, an in-depth analysis of its sensitivity is carried out for different signal lengths, noisy environments, and quasi-stationary conditions. Then, its robustness is evaluated for different Total Harmonic Distortion (THD) values. After that, the classifier computation complexity is analyzed. Finally, a comparison is carried out between the proposed technique and two other classifiers, namely the symmetrical components and the three-phase three-angle algorithms.

The main contributions of this paper, with respect to the abovediscussed literature, can be summarized as follows:

- Compared to the classifiers published in [18], [19], [21], the proposed algorithm has the ability to provide a complete sag and swell classification. In contrast to pattern recognition-based classifiers, the proposed algorithm does not require any training database and no parameter to be set. Furthermore, while most of the PQ disturbance classification techniques exploit single-phase information, the proposed classifier will exploit three-phase information that allow better classification performances [29].

- A comparison between two proposed pre-classifiers based on Information Theoretical Criteria has been made. The first preclassifier is called the Maximum Likelihood (ML) pre-classifier and the seconde one is called the Approximate (App) preclassifier. The former yields the highest statistical performances, while the latter has a lower computation complexity. Then, a classifier based on estimated symmetrical component values has been proposed and evaluated. It allows the classification of the corresponding sag or swell.

- An experimental validation with time-varying real power system signals is carried out.

This paper is organized as follows. Section II presents the signal and phasor models. Section III describes the classification method for sags and swells signatures, then Section IV illustrates the effectiveness of the proposed technique evaluated using Monte Carlo simulations on synthetic signals and using real power system data.

\section{Signal AND Phasor MOdELS}

This section presents the three-phase signal and phasor models. Based on the phasor model, we also describe the 4-classes and the nine sags and swells signatures.

\section{A. Three-Phase Signals Model}

In a three-phase power system, the signal on phase $m(m=0,1,2)$ can be expressed as [39]

$$
x_{m}[n]=a_{m} \cos \left(n \omega_{0}+\varphi_{m}\right)+b_{m}[n],
$$

where $a_{m}$ and $\varphi_{m}$ correspond to the amplitude and phase angle, respectively, $\omega_{0}$ denotes the normalized angular frequency, and $b_{m}[n]$ refers to the additive noise. The parameters $a_{m}$ and $\varphi_{m}$ are usually described more compactly by introducing the complex phasor. Mathematically, the complex phasor on phase $m$ is defined as

$$
c_{m} \triangleq a_{m} e^{j \varphi_{m}}
$$

Without loss of generality, we assume that the voltage sensors record $N$ consecutive samples $(n=0,1 \cdots, N-1)$. By using a matrix notation, the recorded samples on the three phases can be written as

$$
\mathbf{X}=\mathbf{A C}+\mathbf{B},
$$

where

- $\mathbf{X}$ and $\mathbf{B}$ are $N \times 3$ matrices containing the recorded and noise samples, respectively. These matrices are defined by

$$
\begin{aligned}
\mathbf{X} & =\left[\begin{array}{ccc}
x_{0}[0] & x_{1}[0] & x_{2}[0] \\
\vdots & \vdots & \vdots \\
x_{0}[N-1] & x_{1}[N-1] & x_{2}[N-1]
\end{array}\right], \\
\mathbf{B} & =\left[\begin{array}{ccc}
b_{0}[0] & b_{1}[0] & b_{2}[0] \\
\vdots & \vdots & \vdots \\
b_{0}[N-1] & b_{1}[N-1] & b_{2}[N-1]
\end{array}\right],
\end{aligned}
$$

- A is a $N \times 2$ real-valued matrix which is defined by

$$
\mathbf{A}=\left[\begin{array}{cc}
1 & 0 \\
\cos \left(w_{0}\right) & \sin \left(w_{0}\right) \\
\vdots & \vdots \\
\cos \left((N-1) w_{0}\right) & \sin \left((N-1) w_{0}\right)
\end{array}\right],
$$


- $\mathbf{C}$ is a $2 \times 3$ real-valued matrix containing the real and imaginary parts of the phasors and is defined by

$$
\mathbf{C}=\left[\begin{array}{ccc}
a_{0} \cos \left(\varphi_{0}\right) & a_{1} \cos \left(\varphi_{1}\right) & a_{2} \cos \left(\varphi_{2}\right) \\
-a_{0} \sin \left(\varphi_{0}\right) & -a_{1} \sin \left(\varphi_{1}\right) & -a_{2} \sin \left(\varphi_{2}\right)
\end{array}\right]
$$

In the following, we resort to a vectorized version of $\mathbf{X}$ to analyse the recorded signal. Let us introduce vec(.) the vectorization operator that converts a matrix into a column vector by stacking the columns of the matrix on top of one another. Using the properties of the vectorization operator, the three-phase signal $\mathbf{x} \triangleq \operatorname{vec}(\mathbf{X})$ can be expressed into a $3 N \times 1$ column vector as

$$
\mathbf{x}=\left(\mathbf{I}_{3} \otimes \mathbf{A}\right) \mathbf{c}+\mathbf{b},
$$

where $\mathbf{b}=\operatorname{vec}(\mathbf{B}), \mathbf{c} \triangleq \operatorname{vec}(\mathbf{C}), \mathbf{I}_{3}$ is the $3 \times 3$ identity matrix, and $\otimes$ corresponds to the Kronecker product.

\section{B. Phasor Model}

In order to analyze the phasors configuration, it is usually convenient to decompose the three complex phasors into a more synthetic basis. In this subsection, we propose to decompose the three phasors $c_{0}, c_{1}$, and $c_{2}$ into a basis composed of 3 symmetrical components called the zero-sequence $z_{0}$, positive-sequence $z_{1}$, and negative-sequence $z_{2}$. The complex phasors $c_{1}, c_{2}$ and $c_{3}$ can be expressed according to these three symmetrical components through the Fortescue transform as [40]

$$
\left[\begin{array}{l}
c_{0} \\
c_{1} \\
c_{2}
\end{array}\right]=\left[\begin{array}{ccc}
1 & 1 & 1 \\
1 & e^{4 j \pi / 3} & e^{2 j \pi / 3} \\
1 & e^{2 j \pi / 3} & e^{4 j \pi / 3}
\end{array}\right]\left[\begin{array}{c}
z_{0} \\
z_{1} \\
z_{2}
\end{array}\right] .
$$

The main benefit of using the symmetrical components relies on the fact that, under nominal conditions, the symmetrical components are sparse i.e. $z_{0}=z_{2}=0$. In this paper, we consider the 4 following classes.

- $\mathcal{C}_{1}$ : Zero and negative sequences are equal to 0 i.e. $z_{0}=z_{2}=0$.

- $\mathcal{C}_{2}$ : Zero sequence is equal to 0 i.e. $z_{0}=0$.

- $\mathcal{C}_{3}$ : Negative sequence is equal to 0 i.e. $z_{2}=0$.

- $\mathcal{C}_{4}$ : All sequences are different from 0.

For the 4 considered classes, the vector containing the real and imaginary parts of the three phasors, $\mathbf{c}$, in (8) can be expressed with respect to the real and imaginary parts of the symmetrical components as

$$
\mathbf{c}=\mathbf{W}_{k} \mathbf{s}_{k},
$$

where the matrix $\mathbf{W}_{k}$ and the augmented symmetrical components $\mathbf{s}_{k}$ depend on the class number. For example, for Class $\mathcal{C}_{1}$, the vector c can be decomposed as

$$
\mathbf{c}=\underbrace{\left[\begin{array}{l}
\mathbf{Q}_{0} \\
\mathbf{Q}_{1} \\
\mathbf{Q}_{2}
\end{array}\right]}_{\mathbf{W}_{1}} \underbrace{\left[\begin{array}{c}
\Re e\left(z_{1}\right) \\
\Im m\left(z_{1}\right)
\end{array}\right]}_{\mathbf{s}_{1}},
$$

where

$$
\mathbf{Q}_{k}=\left[\begin{array}{cc}
\Re e\left(e^{2 j k \pi / 3}\right) & \Im m\left(e^{2 j k \pi / 3}\right) \\
\Im m\left(e^{2 j k \pi / 3}\right) & -\Re e\left(e^{2 j k \pi / 3}\right)
\end{array}\right] .
$$

Table I presents the expressions of $\mathbf{W}_{k}$ and $\mathbf{s}_{k}$ for the classes $\mathcal{C}_{1}, \mathcal{C}_{2}$, $\mathcal{C}_{3}$, and $\mathcal{C}_{4}$.

Note that the proposed classification is also related to the commonly used ABC classification [41]. The ABC classification is composed of nine sag/swell signatures, denoted with letters from $\mathrm{A}$ to I [23], [41]. These signatures are characterized by a complex prefault voltage, $E$, and a complex post-fault voltage, $V$ [41]. For each
TABLE I

EXPRESSIONS OF $\mathbf{W}_{k}$ AND $\mathbf{S}_{k}$ WITH RESPECT TO CLASS $\mathcal{C}_{k}$.

\begin{tabular}{|c|c|c|c|c|}
\hline Class & $\mathcal{C}_{1}$ & $\mathcal{C}_{2}$ & $\mathcal{C}_{3}$ & $\mathcal{C}_{4}$ \\
\hline $\mathrm{k}$ & 1 & 2 & 3 & 4 \\
\hline $\mathbf{W}_{k}$ & {$\left[\begin{array}{l}\mathbf{Q}_{0} \\
\mathbf{Q}_{1} \\
\mathbf{Q}_{2}\end{array}\right]$} & {$\left[\begin{array}{ll}\mathbf{Q}_{0} & \mathbf{Q}_{0} \\
\mathbf{Q}_{1} & \mathbf{Q}_{2} \\
\mathbf{Q}_{2} & \mathbf{Q}_{1}\end{array}\right]$} & {$\left[\begin{array}{ll}\mathbf{Q}_{0} & \mathbf{Q}_{0} \\
\mathbf{Q}_{0} & \mathbf{Q}_{1} \\
\mathbf{Q}_{0} & \mathbf{Q}_{2}\end{array}\right]$} & {$\left[\begin{array}{lll}\mathbf{Q}_{0} & \mathbf{Q}_{0} & \mathbf{Q}_{0} \\
\mathbf{Q}_{0} & \mathbf{Q}_{1} & \mathbf{Q}_{2} \\
\mathbf{Q}_{0} & \mathbf{Q}_{2} & \mathbf{Q}_{1}\end{array}\right]$} \\
\hline $\mathbf{s}_{k}$ & {$\left[\begin{array}{c}\Re e\left(z_{1}\right) \\
\Im m\left(z_{1}\right)\end{array}\right]$} & {$\left[\begin{array}{c}\Re e\left(z_{1}\right) \\
\Im m\left(z_{1}\right) \\
\Re e\left(z_{2}\right) \\
\Im m\left(z_{2}\right)\end{array}\right]$} & {$\left[\begin{array}{c}\Re e\left(z_{0}\right) \\
\Im m\left(z_{0}\right) \\
\Re e\left(z_{1}\right) \\
\Im m\left(z_{1}\right)\end{array}\right]$} & {$\left[\begin{array}{c}\Re e\left(z_{0}\right) \\
\Im m\left(z_{0}\right) \\
\Re e\left(z_{1}\right) \\
\Im m\left(z_{1}\right) \\
\Re e\left(z_{2}\right) \\
\Im m\left(z_{2}\right)\end{array}\right]$} \\
\hline
\end{tabular}
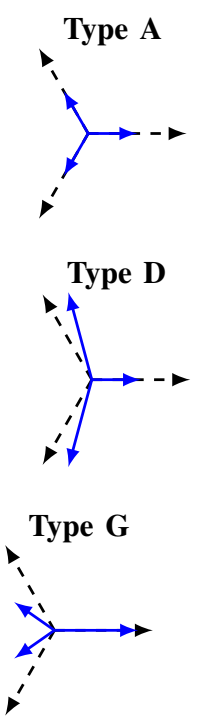
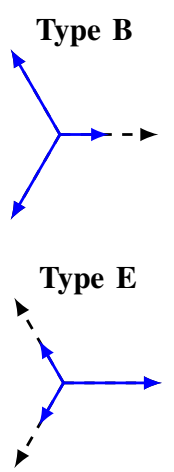

Type $\mathbf{H}$

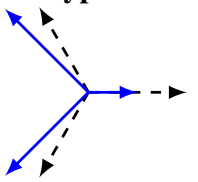

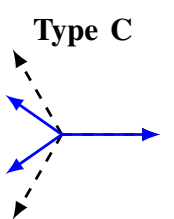

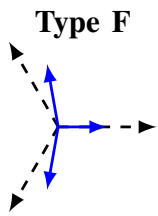

Type I

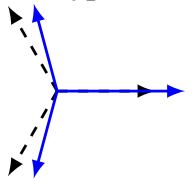

Fig. 1. ABC Classification: Sags and swells signatures [23].

TABLE II

Link between the Proposed and ABC Classifications.

\begin{tabular}{|c|c|c|c|c|}
\hline Type & $z_{0}$ & $z_{1}$ & $z_{2}$ & Class \\
\hline Balanced & 0 & $E$ & 0 & $\mathcal{C}_{1}$ \\
$\mathrm{~A}$ & 0 & $V$ & 0 & \\
\hline $\mathrm{C}$ & 0 & $\frac{V+E}{2+}$ & $\frac{E-V}{V-E}$ & \\
$\mathrm{D}$ & 0 & $\frac{V+E}{2}$ & $\frac{V-E}{2}$ & $\mathcal{C}_{2}$ \\
$\mathrm{~F}$ & 0 & $\frac{2 V+E}{3}$ & $\frac{V-E}{3}$ & \\
$\mathrm{G}$ & 0 & $\frac{2 V^{3}+E}{3}$ & $\frac{E-V}{3}$ & \\
\hline $\mathrm{H}$ & $V-E$ & $E$ & 0 & $\mathcal{C}_{3}$ \\
$\mathrm{I}$ & $\frac{3(E-V)}{2}$ & $E$ & 0 & \\
\hline $\mathrm{B}$ & $\frac{V-E}{3}$ & $\frac{V+2 E}{3}$ & $\frac{V-E}{3}$ & \multirow{2}{*}{$\mathcal{C}_{4}$} \\
$\mathrm{E}$ & $\frac{E-V}{3}$ & $\frac{2 V^{3}+E}{3}$ & $\frac{E-V}{3}$ & \\
\hline
\end{tabular}

signature, the phasor configuration is illustrated in Fig. 1, where the dashed and solid arrows present the phasor configuration before and after voltage sag/swell, respectively. The link between the proposed and $\mathrm{ABC}$ classifications is presented in Table II. In particular, one can note that the proposed classification can be used to pre-classify the nine signatures of the $\mathrm{ABC}$ classification.

\section{General Vectorized Signal Model}

This section presents the general vectorized signal model. Using (8) and (10), the signal model can be described under the general form

$$
\mathbf{x}=\mathbf{M}_{k} \mathbf{s}_{k}+\mathbf{b}
$$




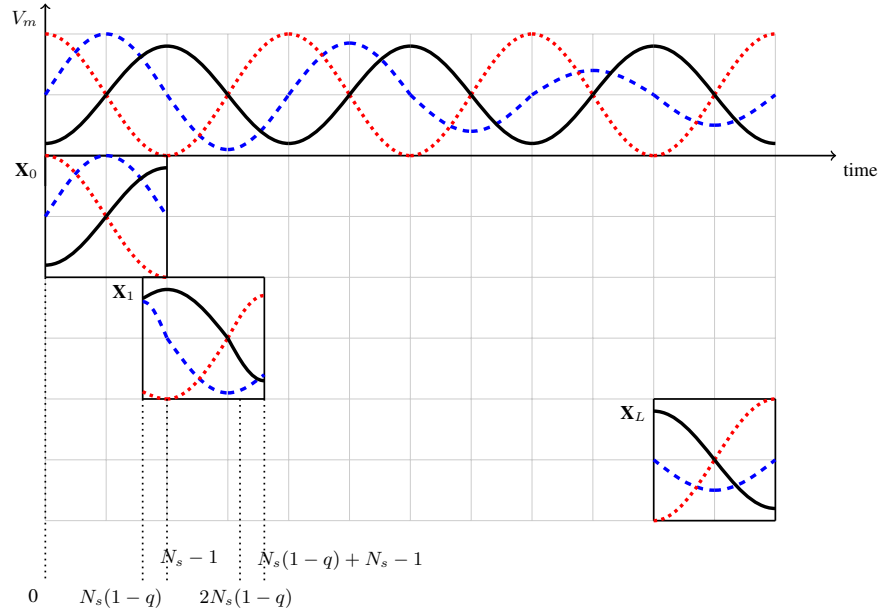

Fig. 2. Concept of sliding window under quasi-stationary conditions.

where $k$ corresponds to the class number and

$$
\mathbf{M}_{k} \triangleq\left(\mathbf{I}_{3} \otimes \mathbf{A}\right) \mathbf{W}_{k} .
$$

The goal of this paper is to determine the phasor configuration, i.e. the class number $k$ and the signature, from the vectorized three-phase signal $\mathbf{x}$.

Note that, while the theoretical methods presented in the following sections are based on the stationary assumption, these methods can be easily extended to the non-stationary case. Indeed, when the signal parameters are time-varying, a straightforward solution is to use a short-time analysis as illustrated by Fig. 2. In Fig. 2, the analysis is performed on several signal blocks with a window length of $N_{s}$ samples. By considering an overlapping parameter $0 \leq q<1$, the $l^{\text {th }}$ signal block can be mathematically expressed by

$$
\mathbf{X}_{l}=\left[\begin{array}{ccc}
x_{0}\left[N_{b}\right] & x_{1}\left[N_{b}\right] & x_{2}\left[N_{b}\right] \\
\vdots & \vdots & \vdots \\
x_{0}\left[N_{e}\right] & x_{1}\left[N_{e}\right] & x_{2}\left[N_{e}\right]
\end{array}\right],
$$

where $N_{b}=l N_{s}(1-q), N_{e}=l N_{s}(1-q)+N_{s}-1$. In this context, the $l^{\text {th }}$ vectorized block, $\mathbf{x}_{l}=\operatorname{vec}\left(\mathbf{X}_{l}\right)$, is given by

$$
\mathbf{x}_{l}=\mathbf{M}_{k} \mathbf{s}_{k l}+\mathbf{b}_{l},
$$

where $\mathbf{s}_{k l}$ corresponds to the $l^{\text {th }}$ augmented time-varying symmetrical components. By using a small window length $N_{s}$, the signal parameters and the class number $k$ can be tracked over time.

\section{Signature Classifier}

This section presents the proposed decision-tree classifier aiming at classifying the signal into one of the nine classes of the $A B C$ classification. Specifically, the first subsection describes the proposed decision tree and the second one focuses on the decision algorithms.

\section{A. Decision Tree}

Fig. 3 depicts the decision tree of the proposed classifier. The proposed decision tree is based on two stages, which are the preclassification into one of the 4 classes $\left(\mathcal{C}_{1}, \mathcal{C}_{2}, \mathcal{C}_{3}\right.$, and $\left.\mathcal{C}_{4}\right)$ and the final classification into one of the nine signatures of the $\mathrm{ABC}$ classification.

In the first stage, as the 4 classes are nested ${ }^{1}$, we propose to reformulate the classification problem as a pure model order selection

${ }^{1}$ For example, the class $\mathcal{C}_{1}$ is a particular case of the class $\mathcal{C}_{3}$ problem. Indeed, Table I shows that the pre-class can be determined from the identification of the model order, i.e. the size of the vector $\mathbf{s}_{k}$. Specifically, the size of the vector $\mathbf{s}_{k}$ is equal to 2 for the class $\mathcal{C}_{1}, 4$ for the classes $\mathcal{C}_{2}$ and $\mathcal{C}_{3}$, and 6 for the class $\mathcal{C}_{4}$. To determine the model order, we propose to use a detection technique based on Information Theoretic Criterion [43].

Once the nine signatures have been pre-classified into one of the 4 classes, the corresponding signature type can be classified by analyzing the symmetrical components. As these components are unknown, we propose to estimate them by using the Maximum Likelihood technique. Then, we determine the signature by analyzing the values of the estimated symmetrical components. Specifically, for $\mathcal{C}_{1}$, it is possible to discriminate between sag type A and balanced power system by comparing the value of $\left|\widehat{z}_{1}\right|$ with the pre-fault value. For $\mathcal{C}_{2}$, the value of $\left|\widehat{z}_{1}+\widehat{z}_{2}\right|$ allows the discrimination between type $\mathrm{C}$ and types $\mathrm{D}, \mathrm{F}$, and $\mathrm{G}$. Then, the discrimination between type $\mathrm{D}$ and types $F$ and $G$ can be obtained by analyzing the value of $\left|\widehat{z}_{1}-\widehat{z}_{2}\right|$. For class $\mathcal{C}_{3}, \Re e\left(\frac{\bar{z}_{0}}{\widehat{z}_{1}}\right)$ allows the classification between types I and $\mathrm{H}$. Finally, for $\mathcal{C}_{4}$, the value $\left|\widehat{z}_{1}-\widehat{z}_{0}\right|$ allows the discrimination between types $\mathrm{B}$ and $\mathrm{E}$. Note that the proposed decision tree is not able to discriminate between types $\mathrm{F}$ and $\mathrm{G}$.

\section{B. Proposed Decision Algorithms}

In this subsection, we show how to pre-classify the signal into 4 classes using Information Theoretical Criteria, and how to estimate the symmetrical components $z_{1}, z_{2}$, and $z_{3}$ using the Maximum Likelihood technique.

Regarding the pre-classifier, the selected class $C_{k}$ based on Information Theoretical Criteria is the one that minimizes the following penalized likelihood function [44]

$$
\widehat{k}=\arg \min _{k=1,2,3,4}-2 \ln p\left(\mathbf{x}, \widehat{\mathbf{s}}_{k}\right)+\gamma_{k},
$$

where $\ln p\left(\mathbf{x}, \widehat{\mathbf{s}}_{k}\right)$ denotes the $\log$-likelihood function of the vectorized signal $\mathbf{x}$ for the class $k$, and $\widehat{\mathbf{s}}_{k}$ corresponds to the Maximum Likelihood Estimator of $\mathbf{s}_{k}$. The term $\gamma_{k}$ is a penalty function that depends on the total number of samples and on the number of free parameters. In the following paragraphs, we provide explicit expressions for the log-likelihood function and penalty term.

1) Expression of the log-likelihood function: Under the assumption that the additive noise is Gaussian distributed with a zero mean and a variance equal to $\sigma^{2}$ i.e. $\mathbf{b} \sim \mathcal{N}\left(\mathbf{0}, \sigma^{2} \mathbf{I}_{3 N}\right)$, it has been demonstrated in [43] that the $\log$-likelihood function $\ln p\left(\mathbf{x}, \widehat{\mathbf{s}}_{k}\right)$ is equal to

$$
-2 \ln p\left(\mathbf{x}, \widehat{\mathbf{s}}_{k}\right)=\mathrm{constant}+3 N \ln \widehat{\sigma}_{k}^{2},
$$

where $\widehat{\sigma}_{k}^{2}$ is the Maximum Likelihood Estimator of the noise variance under the assumption that the signal comes from class $\mathcal{C}_{k}$. Note that, in addition to the model order, the estimator of the noise variance $\widehat{\sigma}^{2}$ also provides a simple measure of the goodness of fit for competing models with the same model order such as $\mathcal{C}_{2}$ and $\mathcal{C}_{3}$. Mathematically, the noise variance estimator is given by

$$
\widehat{\sigma}_{k}^{2}=\frac{1}{3 N}\left\|\mathbf{x}-\mathbf{M}_{k} \widehat{\mathbf{s}}_{k}\right\|^{2},
$$

where $\widehat{\mathbf{s}}_{k}$ corresponds to the Maximum Likelihood Estimator of the vector $\mathbf{s}_{k}$. As $\widehat{\mathbf{s}}_{k}=\left(\mathbf{M}_{k}^{T} \mathbf{M}_{k}\right)^{-1} \mathbf{M}_{k}^{T} \mathbf{x}$, it follows that

$$
\widehat{\sigma}_{k}^{2}=\mathbf{x}^{T} \mathbf{P}_{k}^{\perp} \mathbf{x},
$$

where $\mathbf{P}_{k}^{\perp}$ is the orthogonal projector onto the null space of $\mathbf{M}_{k}$, which is defined as [45]

$$
\mathbf{P}_{k}^{\perp} \triangleq \mathbf{I}_{3 N}-\mathbf{M}_{k}\left(\mathbf{M}_{k}^{T} \mathbf{M}_{k}\right)^{-1} \mathbf{M}_{k}^{T} .
$$






Fig. 3. Flowchart of the proposed classification algorithm.

It is worth mentioning that the inverse of $\mathbf{M}_{k}^{T} \mathbf{M}_{k}$ is generally difficult to obtain analytically. Nevertheless, in some particular cases, this inverse has a simple closed form. Indeed, when the number of samples is large, i.e $N \gg 1$ or when $N$ is a multiple of a half of the signal period, i.e. $N=k \pi / \omega_{0}$, it can be checked that $\mathbf{A}^{T} \mathbf{A}=\frac{N}{2} \mathbf{I}$. Furthermore, as $\mathbf{W}_{k}^{T} \mathbf{W}_{k}=3 \mathbf{I}$, it follows that $\mathbf{M}_{k}^{T} \mathbf{M}_{k} \approx \frac{3 N}{2} \mathbf{I}$. Therefore, the orthogonal projector, $\mathbf{P}_{k}^{\perp}$, can be approximated by

$$
\mathbf{P}_{k}^{\perp} \approx \mathbf{I}_{3 N}-\frac{2}{3 N} \mathbf{M}_{k} \mathbf{M}_{k}^{T} .
$$

Based on the values of $\mathbf{P}_{k}^{\perp}$, we propose two different pre-classifiers: the Maximum Likelihood (ML) classifiers and the Approximate (App) one. The ML classifier is obtained by using the exact value of the orthogonal projector (21) in (20), (18), and (17). The App classifier is obtained by using the approximated projector (22) in (20), (18), and (17). Finally for the ML and App classifiers, the estimated classes are respectively given by

$$
\begin{aligned}
& \widehat{k}^{M L}=\arg \min _{k=1,2,3,4} 3 N \ln \left(\mathbf{x}^{T} \mathbf{P}_{k}^{\perp} \mathbf{x}\right)+\gamma_{k} \\
& \widehat{k}^{A p p}=\arg \min _{k=1,2,3,4} 3 N \ln \left(\|\mathbf{x}\|^{2}-\frac{2}{3 N}\left\|\mathbf{M}_{k}^{T} \mathbf{x}\right\|^{2}\right)+\gamma_{k},
\end{aligned}
$$

where $\|\mathbf{x}\|^{2} \triangleq \mathbf{x}^{T} \mathbf{x}$ corresponds to the squared norm of $\mathbf{x}$. Note that the App pre-classifier has a lower computational complexity than the ML pre-classifier since it requires a smaller number of matrix multiplications and does not involve any matrix inversion.

2) Expression of $\gamma_{k}$ : The goal of the penalty term $\gamma_{k}$ is to promote simplicity over complexity. Mathematically, this function penalizes the log-likelihood function with respect to the number of samples, $3 N$, and estimated parameters, $n_{k}$. Several penalty terms have been proposed in the literature based on different motivations [43]. In particular, the most commonly used penalty terms are the Akaike Information Criterion (AIC) $\gamma_{k}^{A I C}=2 n_{k}$, and the Bayesian Information Criterion (BIC) $\gamma_{k}^{B I C}=n_{k} \ln (3 N)$. For the class $k$, the number of estimated parameters is equal to $n_{k}=l_{k}+1$, where $l_{k}$
TABLE III

Penalty Function With RESPECT to Class $\mathcal{C}_{k}$

\begin{tabular}{|c|c|c|c|c|}
\hline Class & $\mathcal{C}_{1}$ & $\mathcal{C}_{2}$ & $\mathcal{C}_{3}$ & $\mathcal{C}_{4}$ \\
\hline$n_{k}$ & 3 & 5 & 5 & 7 \\
\hline$\gamma_{k}^{A I C}$ & 6 & 10 & 10 & 14 \\
$\gamma_{k}^{B I C}$ & $3 \ln (3 N)$ & $5 \ln (3 N)$ & $5 \ln (3 N)$ & $7 \ln (3 N)$ \\
\hline
\end{tabular}

corresponds to the size of $\widehat{\mathbf{s}}_{k}$ and the number 1 is due to the noise variance estimation. Finally, the values of the AIC and BIC criteria are summed up in Table III for classes $\mathcal{C}_{1}, \mathcal{C}_{2}, \mathcal{C}_{3}$, and $\mathcal{C}_{4}$.

The second stage of the proposed classifier uses the symmetrical component estimates to determine the signature type. Statistically, the ML estimator of $\widehat{z}_{0}, \widehat{z}_{1}$, and $\widehat{z}_{2}$ can be easily extracted from the ML estimator of $\mathbf{s}_{k}$. The exact and approximate estimators of $\mathbf{s}_{k}$ are respectively given by [44]

$$
\begin{aligned}
\widehat{\mathbf{s}}_{k}^{M L} & =\left(\mathbf{M}_{k}^{T} \mathbf{M}_{k}\right)^{-1} \mathbf{M}_{k}^{T} \mathbf{x} \\
\widehat{\mathbf{s}}_{k}^{A p p} & =\frac{2}{3 N} \mathbf{M}_{k}^{T} \mathbf{x} .
\end{aligned}
$$

Then, the expression of the $v^{t h}$ symmetrical component, denoted $\widehat{z}_{v}$, can be obtained from $\widehat{\mathbf{s}}_{k}^{M L}$, or $\widehat{\mathbf{s}}_{k}^{A p p}$, by constructing the complex value

$$
\widehat{z}_{v}=\left[\widehat{\mathbf{s}}_{k}\right]_{2 u}+j\left[\widehat{\mathbf{s}}_{k}\right]_{2 u+1},
$$

where $\left[\widehat{\mathbf{s}}_{k}\right]_{u}$ corresponds to the $u^{t h}$ element of $\widehat{\mathbf{s}}_{k}$. Note that the index $v$ depends on the class $\mathcal{C}_{k}$. Indeed using Table I, it can be readily checked that $v=1$ when $u=0$ for class $\mathcal{C}_{1}, v=u+1$ for class $\mathcal{C}_{2}, v=u$ for classes $\mathcal{C}_{3}$ and $\mathcal{C}_{4}$.

\section{Simulation and Experimental Results}

In this section the performance of the proposed classifier illustrated in Fig. 3 is evaluated through synthetics signals and real power 
TABLE IV

4-Classes: Simulation Parameters.

\begin{tabular}{|c|c|c|c|c|}
\hline & $\mathcal{C}_{1}$ & $\mathcal{C}_{2}$ & $\mathcal{C}_{3}$ & $\mathcal{C}_{4}$ \\
\hline$c_{0}$ & $0.5 \angle-20^{\circ}$ & $1 \angle-20^{\circ}$ & $0.5 \angle-20^{\circ}$ & $1 \angle-20^{\circ}$ \\
\hline$c_{1}$ & $0.5 \angle-140^{\circ}$ & $0.66 \angle-159.10^{\circ}$ & $1.32 \angle-159.10^{\circ}$ & $0.5 \angle-140^{\circ}$ \\
\hline$c_{2}$ & $0.5 \angle 100^{\circ}$ & $0.66 \angle 119.11^{\circ}$ & $1.32 \angle 119.11^{\circ}$ & $0.5 \angle 100^{\circ}$ \\
\hline
\end{tabular}

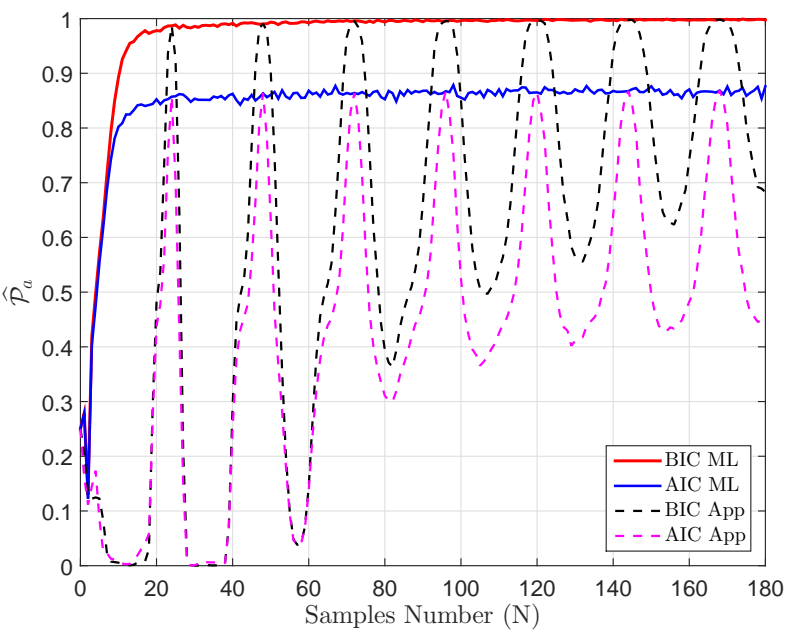

Fig. 4. Average probability of correct classification versus samples number for $S N R=15 \mathrm{~dB}$.

system data. Two classifiers have been implemented: the Maximum Likelihood (ML) classifier composed of the exact ML pre-classifier in (23) and the ML estimator of $\widehat{z}_{v}$ in (25), and the approximate (App) classifier composed of the approximate pre-classifier in (24) and the approximate ML estimator of $\widehat{z}_{v}$ in (26).

\section{A. Monte Carlo Simulation Results}

In this subsection, the two pre-classifiers are evaluated using synthetic signals. The classification performance, which is quantified through the average probability of correct classification, is evaluated using both the AIC and BIC penalty terms. The average probability of correct classification is estimated using $N_{m c}=1000$ Monte Carlo trials for each class $\mathcal{C}_{k}$. In each simulation, the signal is generated from the model defined in (1) with $\omega_{0}=2 \pi f_{0} / F_{s}$, where $f_{0}=50 \mathrm{~Hz}$ and $F_{s}=48 \times f_{0}=2400 \mathrm{~Hz}$. The amplitudes and initial phases of the three complex phasors for 4-classes are given in Table IV. Then, the average probability of correct classification is estimated as follows

$$
\widehat{\mathcal{P}}_{a}=\frac{1}{4 N_{m c}} \sum_{k=1}^{4} \sum_{n=1}^{N_{c}} \delta\left(k-\widehat{k}_{n}\right)
$$

where $\widehat{k}_{n}$ is the estimated class for the $n^{\text {th }}$ trial, and $\delta(l)$ is the Kronecker delta which is equal to 1 if $l=1$ and zero elsewhere. In the next subsections, the average probability of correct classification is analyzed for different signal lengths, $N$, and Signal to Noise Ratio $(S N R)$, where the $S N R$ is defined as

$$
\eta=\frac{1}{6 \sigma^{2}} \sum_{k=0}^{2} a_{k}^{2}
$$

and $\sigma^{2}$ corresponds to the noise variance.

1) Probability of correct classification versus number of samples: Fig. 4 shows the influence of the number of samples on the average probability of correct classification when the $S N R$ is equal to $15 \mathrm{~dB}$. It can be observed that the ML pre-classifier outperforms, in most of

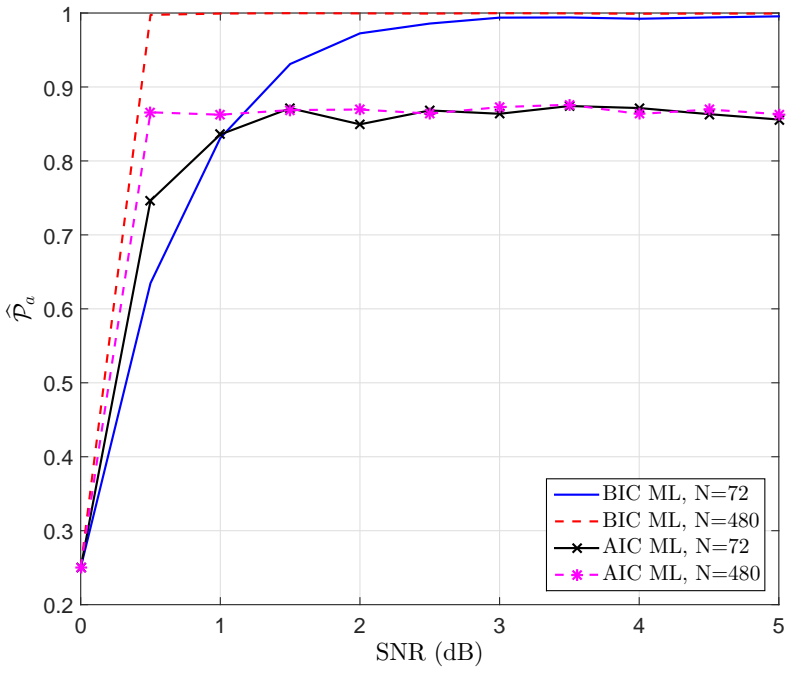

Fig. 5. Average probability of correct classification versus $S N R$ for $N=$ 72 and $N=480$.

the cases, the approximate one. Nevertheless, these two pre-classifiers lead to the same probability of correct classification when the number of samples is large or when $N$ is a multiple of a half cycle i.e. $N=(48 / 2) k=24 k$. It is important to note that phasor measurement units (PMUs) can measure 50/60 Hz AC waveforms (voltages and currents) typically at a rate of a multiple of a cycle (48 samples per cycle).

Regarding the penalty factors, we observe that the pre-classifier using BIC penalty term outperforms the pre-classifier using AIC penalty term whatever the signal length is, for the ML and App preclassifiers. In particular, we can note that for large $N$ the BIC penalty term leads to an average probability of correct classification equal to 1, while the AIC penalty term leads to a smaller average probability. For small $N$, we also note that the BIC has a faster response time than the AIC penalty term.

2) Probability of correct classification versus noise variance: Fig. 5 highlights the influence of the $S N R$ on the average probability of correct classification $\left(\widehat{\mathcal{P}}_{a}\right)$. In this simulation, the number of samples $N$ is set to 72 and 480 samples (multiple of a half-cycle). Under these conditions, the ML and approximate pre-classifiers lead to the same probability of correct classification. For $N=72$ samples, the BIC ML pre-classifier leads to $\widehat{\mathcal{P}}_{a}=1$ when the $S N R$ is greater than $3 \mathrm{~dB}$. For $N=480$ samples, the BIC ML pre-classifier leads to $\widehat{\mathcal{P}}_{a}=1$ when $S N R$ is greater than $0.5 \mathrm{~dB}$. This clearly shows that the proposed pre-classifier has good capabilities under a highly noisy environment. For the AIC pre-classifier, we observe that $\widehat{\mathcal{P}}_{a}$ is not equal to 1 even for large $S N R s$ and $N$. To explain this limitation, Tables $\mathrm{V}$ and VI present the confusion matrices when $N=480$ samples and at $S N R=5 \mathrm{~dB}$. For the ML pre-classifier using BIC penalty term, we observe that each trial is correctly classified since the confusion matrix only contains diagonal elements. For the ML preclassifier using the AIC penalty term, we observe that the confusion matrix also contains non-diagonal elements and is upper triangular. The upper triangular structure shows that the AIC penalty term seems to overestimate the model order.

3) Robustness of the proposed classifier: This subsection evaluates the performance of the proposed algorithm under harmonic environments. By introducing harmonic components in (1), the signal model 
TABLE V

AIC CRITERION: ML PRE-Classifier Confusion MATRIX.

\begin{tabular}{|c|c|c|c|c|}
\hline & $\mathcal{C}_{1}$ & $\mathcal{C}_{2}$ & $\mathcal{C}_{3}$ & $\mathcal{C}_{4}$ \\
\hline $\mathcal{C}_{1}$ & 741 & 135 & 124 & 0 \\
\hline $\mathcal{C}_{2}$ & 0 & 874 & 0 & 126 \\
\hline $\mathcal{C}_{3}$ & 0 & 0 & 861 & 147 \\
\hline $\mathcal{C}_{4}$ & 0 & 0 & 0 & 1000 \\
\hline
\end{tabular}

TABLE VI

BIC CRITERION: ML PRE-CLASSIFIER CONFUSION MATRIX.

\begin{tabular}{|c|c|c|c|c|}
\hline & $\mathcal{C}_{1}$ & $\mathcal{C}_{2}$ & $\mathcal{C}_{3}$ & $\mathcal{C}_{4}$ \\
\hline $\mathcal{C}_{1}$ & 999 & 0 & 1 & 0 \\
\hline $\mathcal{C}_{2}$ & 0 & 1000 & 0 & 0 \\
\hline $\mathcal{C}_{3}$ & 0 & 0 & 1000 & 0 \\
\hline $\mathcal{C}_{4}$ & 0 & 0 & 0 & 1000 \\
\hline
\end{tabular}



Fig. 6. Harmonic effect on the proposed classifier. Comparison between $\mathrm{ML}$ classifiers using BIC and AIC penalty terms.

can be described by

$$
\begin{aligned}
x_{m}[n] & =a_{m} \cos \left(n w_{0}+\phi_{m}\right) \\
& +\sum_{h=3,5,7 \ldots} \alpha\left(a_{m h} \cos \left(h n w_{0}+\phi_{m h}\right)\right)+b_{m}[n],
\end{aligned}
$$

where $a_{m h}$ and $\phi_{m h}$ correspond to the amplitude and initial phase of the $h^{\text {th }}$ order harmonic, respectively. The quantity $\alpha>0$ corresponds to the harmonic parameter. The harmonic amplitudes are set to $a_{m 5}=0.06 \mathrm{pu}, a_{m 7}=0.05 \mathrm{pu}, a_{m 11}=0.015 \mathrm{pu}, a_{m 11}=0.03$ $\mathrm{pu}$, and $a_{m 13}=0.03 \mathrm{pu}$.

Fig. 6 shows the $\widehat{\mathcal{P}}_{a}$ of the proposed pre-classifiers versus the Total Harmonic Distortion (THD) for $N=144$ samples and $S N R=$ $5 \mathrm{~dB}$. The Total Harmonic Distortion is controlled by varying the different harmonics amplitudes through the scalar $\alpha$. We show that the performances of the proposed pre-classifiers decreases for a high value of THD. Furthermore, we observe that for a THD less than $50 \%$ the BIC ML pre-classifier leads to a constant $\widehat{\mathcal{P}}_{a}=1$, while for the AIC pre-classifier it is increasing with respect to the THD. For a THD $>70 \%$, the $\widehat{\mathcal{P}}_{a}$ of both pre-classifiers is decreasing when the THD increases due to the model mismatch.

4) Computation complexity: Fig. 7 compares the computation complexity (in seconds) between the ML and App classifiers. The computation time are evaluated by averaging the execution time through 1000 Monte Carlo trials. Results show that both classifiers computation times increase proportionally to the number of samples

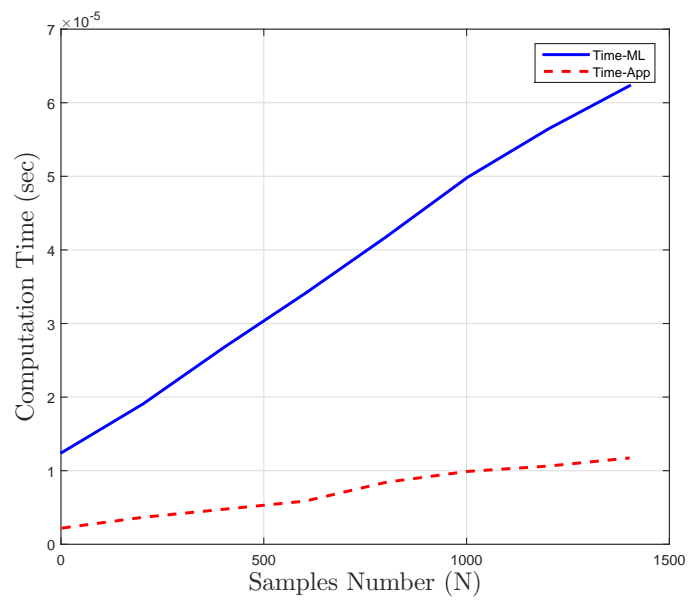

Fig. 7. Computation time versus $N$ : Comparison between $\mathrm{ML}$ and Approximate classifiers.

TABLE VII

Voltage Sags Type C: Comparison of the Proposed Classifier WITH SC AND TP-TA ALGORITHMS

\begin{tabular}{|c|c|c|c|}
\hline Case & $\mathbf{C}$ & $\mathbf{C}$ & $\mathbf{C}$ \\
\hline$\widehat{\mathbf{V}}_{a}$ & $0.85 \angle-20^{\circ}$ & $0.85 \angle-20^{\circ}$ & $0.85 \angle-20^{\circ}$ \\
\hline$\widehat{\mathbf{V}}_{b}$ & $0.56 \angle-118^{\circ}$ & $0.46 \angle-110^{\circ}$ & $0.65 \angle-168^{\circ}$ \\
\hline$\widehat{\mathbf{V}}_{c}$ & $0.56 \angle 160^{\circ}$ & $0.65 \angle 168^{\circ}$ & $0.46 \angle 110^{\circ}$ \\
\hline SCA & $\mathbf{D}_{c}$ & $\mathbf{D}_{c}$ & $\mathbf{C}_{a}$ \\
\hline TP-TAA & $\mathbf{C}_{a}$ & $\mathbf{C}_{a}$ & $\mathbf{D}_{c}$ \\
\hline BIC ML & $\mathbf{C}$ & $\mathbf{C}$ & $\mathbf{C}$ \\
\hline
\end{tabular}

$N$, while having different slopes. Specifically, the slope of the App classifier is lower than the ML classifier one. Therefore, when the signal length $N$ is a multiple of a half-cycle, the approximate classifier is an attractive choice for pre-classification since it has a lower computational cost and reaches the statistical performance of the ML classifier.

5) Classification comparison: This subsection proposes a comparison of the ML classifier using the BIC penalty term (BIC-ML) with two others techniques, namely the symmetrical components and the three-phase three-angle algorithms (SCA and TP-TAA, respectively). The SCA and TP-TAA allow identifying the six voltage sags types among the $\mathrm{C}$ and $\mathrm{D}$ types. For the $\mathrm{SC}$ algorithm, the angle between the drop in positive- and negative-sequence voltages is used for classification [18]. The TP-TA algorithm is based on the remains rms voltage (RV), the inverse remains voltage (IRV), the delta inverse remains voltage $(\triangle \mathrm{IRV})$, and the three angles $\alpha, \beta$, and $\gamma$ [21]. In the following, the performances of the classification algorithms are evaluated using the phasor configurations presented in [21]. For each technique, the phasors are estimated using the ML estimator in (25) with a half-cycle window length.

From Tables VII, VIII, IX, and X, we observe that the proposed classifier (BIC-ML) leads to an accurate classification of voltage sags in all cases, while the SC and TP-TA algorithms exhibit erroneous classification under large-phase angle jump and amplitude. These results show that the proposed algorithm has higher classification performance than the SC and TP-TA algorithms.

\section{B. Experimental Tests}

In this subsection, the performances of the ML classifiers using BIC and AIC penalty terms are evaluated using real power system 
TABLE VIII

Voltage Sags Type C: Comparison of the Proposed Classifier WITH SC AND TP-TA ALGORITHMS

\begin{tabular}{|c|c|c|c|}
\hline Case & $\mathbf{C}$ & $\mathbf{C}$ & $\mathbf{C}$ \\
\hline$\widehat{\mathbf{V}}_{a}$ & $0.85 \angle-20^{\circ}$ & $0.85 \angle-20^{\circ}$ & $0.92 \angle 0^{\circ}$ \\
\hline$\widehat{\mathbf{V}}_{b}$ & $0.78 \angle-177^{\circ}$ & $0.65 \angle-168^{\circ}$ & $0.80 \angle-122^{\circ}$ \\
\hline$\widehat{\mathbf{V}}_{c}$ & $0.34 \angle 104^{\circ}$ & $0.46 \angle 110^{\circ}$ & $0.80 \angle 123^{\circ}$ \\
\hline SCA & $\mathbf{C}_{a}$ & $\mathbf{D}_{c}$ & $\mathbf{C}_{a}$ \\
\hline TP-TAA & $\mathbf{C}_{a}$ & $\mathbf{C}_{a}$ & $\mathbf{C}_{a}$ \\
\hline BIC ML & $\mathbf{C}$ & $\mathbf{C}$ & $\mathbf{C}$ \\
\hline \multicolumn{4}{|r}{} \\
\hline
\end{tabular}

TABLE IX

Voltage Sags Type D: Comparison of the Proposed Classifier WITH SC AND TP-TA ALGORITHMS

\begin{tabular}{|c|c|c|c|}
\hline Case & $\mathbf{D}$ & $\mathbf{D}$ & $\mathbf{D}$ \\
\hline$\widehat{\mathbf{V}}_{a}$ & $0.87 \angle-20^{\circ}$ & $0.10 \angle 84.29^{\circ}$ & $0.70 \angle 0^{\circ}$ \\
\hline$\widehat{\mathbf{V}}_{b}$ & $0.40 \angle-131^{\circ}$ & $0.81 \angle-90.71^{\circ}$ & $0.92 \angle-114^{\circ}$ \\
\hline$\widehat{\mathbf{V}}_{c}$ & $0.82 \angle 133^{\circ}$ & $0.91 \angle 90.63^{\circ}$ & $0.92 \angle 110^{\circ}$ \\
\hline SCA & $\mathbf{D}_{b}$ & $\mathbf{D}_{a}$ & $\mathbf{D}_{a}$ \\
\hline TP-TAA & $\mathbf{D}_{b}$ & $\mathbf{D}_{a}$ & $\mathbf{C}_{b}$ \\
\hline BIC ML & $\mathbf{D}$ & $\mathbf{D}$ & $\mathbf{D}$ \\
\hline
\end{tabular}

TABLE $X$

Voltage Sags Type C and D: Comparison of the Proposed CLASSIFIER WITH SC AND TP-TA ALGORITHMS

\begin{tabular}{|c|c|c|c|}
\hline Case & $\mathbf{C}$ & $\mathbf{D}$ & $\mathbf{D}$ \\
\hline$\widehat{\mathbf{V}}_{a}$ & $1 \angle 0.6^{\circ}$ & $0.92 \angle 06^{\circ}$ & $0.70 \angle 0^{\circ}$ \\
\hline$\widehat{\mathbf{V}}_{b}$ & $0.81 \angle-152^{\circ}$ & $0.92 \angle-130^{\circ}$ & $0.92 \angle-114^{\circ}$ \\
\hline$\widehat{\mathbf{V}}_{c}$ & $0.47 \angle 126^{\circ}$ & $0.70 \angle 120^{\circ}$ & $0.92 \angle 110^{\circ}$ \\
\hline SCA & $\mathbf{C}_{a}$ & $\mathbf{D}_{c}$ & $\mathbf{D}_{a}$ \\
\hline TP-TAA & $\mathbf{C}_{a}$ & $\mathbf{C}_{a}$ & $\mathbf{C}_{b}$ \\
\hline BIC ML & $\mathbf{C}$ & $\mathbf{D}$ & $\mathbf{D}$ \\
\hline
\end{tabular}

data obtained from the DOE/EPRI National Database of Power System Events [46]. The disturbances used in this subsection correspond to the events $2825,2827,2802,2786$, and 2911. These disturbance events correspond to different voltage sag and swell configurations. Regarding the classifier settings, the window length was set to a half-cycle (64 samples) with a half-cycle overlap. Figs. 8-12 present the three-phase signal, the amplitude of the estimated phasors, the selected pre-class and the estimated signature for the 5 events.

For event 1 (Fig. 8), we observe that both pre-classifiers using the BIC and the AIC penalty terms achieve the same results. Indeed, both pre-classifiers lead to null zero- and negative-sequences $\left(\mathcal{C}_{1}\right)$ under balanced conditions. Under transient conditions a non-null symmetrical sequence is obtained $\left(\mathcal{C}_{4}\right)$. It seems that both pre-classifiers can achieve a good classification under balanced or transient conditions. For event 2 (Fig. 9), both pre-classifiers achieve the same results under balanced conditions since they lead to null zero- and negativesequences $\left(\mathcal{C}_{1}\right)$. Under transient conditions, these pre-classifiers give different classification results. The BIC pre-classifier leads to a null zero-sequence $\left(\mathcal{C}_{2}\right)$, while the AIC pre-classifier leads to a nonnull symmetrical-sequence $\left(\mathcal{C}_{4}\right)$. It is observed that after $0.12 \mathrm{~s}$, the ML classifier using the AIC penalty term seems to give incorrect classification since it leads to a type FG instead of type A. In contrast, the ML classifier using the BIC penalty term seems to achieve good classification results. For event 3 (Fig. 10), we observe that both pre-classifiers achieve different classification results. Under balanced conditions, the pre-classifier using the BIC penalty term leads to null zero- and negative-sequences $\left(\mathcal{C}_{1}\right)$, whereas the pre-
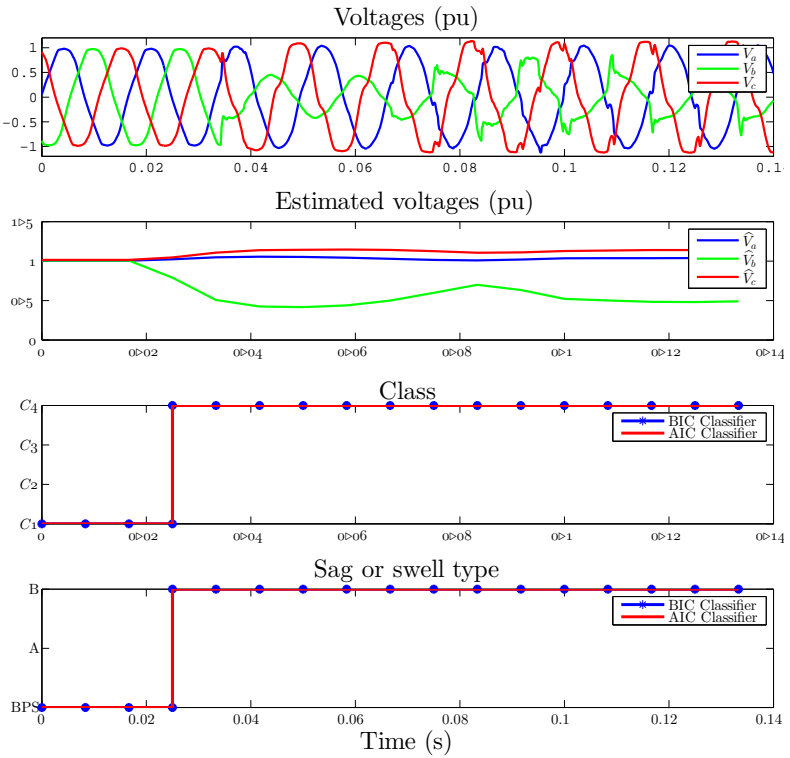

Fig. 8. Event 1 (2827): Voltage sag and swell classification. Comparison between ML classifiers using BIC and AIC penalty terms.
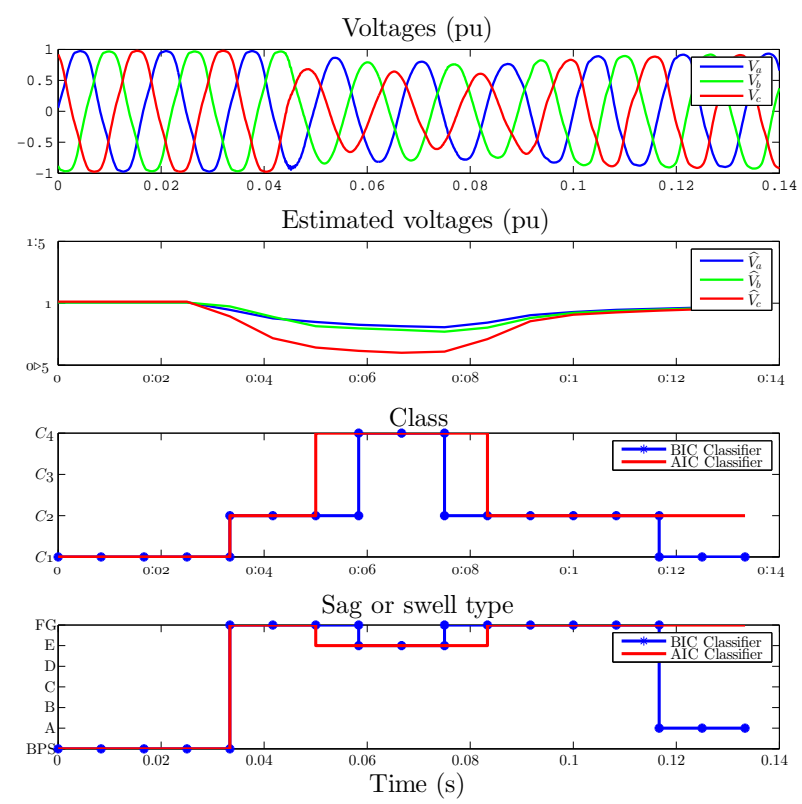

Fig. 9. Event 2 (2825): Voltage sag and swell classification. Comparison between ML classifiers using BIC and AIC penalty terms.

classifier using the AIC penalty term leads to a null zero-sequence $\left(\mathcal{C}_{2}\right)$. Under transient conditions, the BIC pre-classifier leads to a null zero-sequence $\left(\mathcal{C}_{2}\right)$ and the AIC pre-classifier leads to non-null symmetrical-sequences $\left(\mathcal{C}_{4}\right)$. We can note that after $0.10 \mathrm{~s}$ both preclassifiers lead to the same result. For event 4 (Fig. 11) both preclassifiers lead to different results under balanced conditions $\left(\mathcal{C}_{1}\right.$ and $\mathcal{C}_{3}$ ) but achieve the same result after $0.03 \mathrm{~s}$. For event 5 (Fig. 12), the two pre-classifiers lead to the same results except between $0.03 \mathrm{~s}$ and $0.04 \mathrm{~s}$. In most of the cases, we observe that the BIC classifier seems to achieve better classification results under balanced and transient conditions than the AIC classifier. 

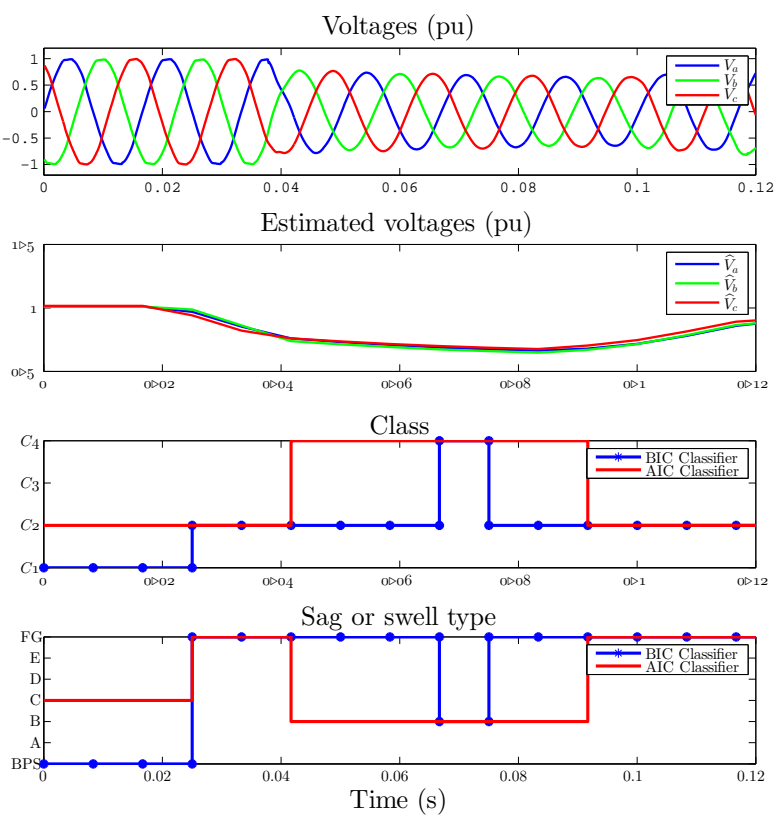

Fig. 10. Event 3 (2802): Voltage sag and swell classification. Comparison between ML classifiers using BIC and AIC penalty terms.

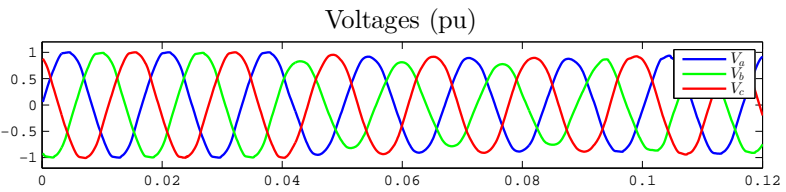

Estimated voltages $(\mathrm{pu})$
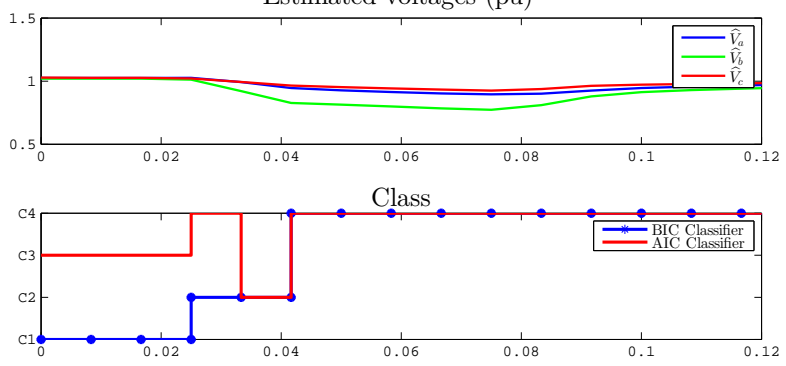

Sag or swell type



Fig. 11. Event 4 (2786): Voltage sag and swell classification. Comparison between ML classifiers using BIC and AIC penalty terms.

\section{Discussion}

The previous sections have focused on the influence of signal length, noise level, harmonics, and window length (overlap) on the performance of the proposed classifiers (ML and approximate classifiers with BIC and AIC penalty terms). Simulation results have clearly shown that the ML classifier using the BIC penalty term achieves higher classification performances whatever the number of samples and SNR are.

The statistical performances of the ML and App classifiers depend on the number of samples, $N$, and on the $S N R$. Specifically, increasing the value of $N$ or $S N R$ also increases the classification performance of the ML classifier. Regarding the differences between the ML and

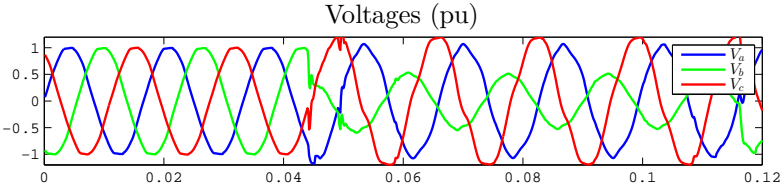

Estimated voltages $(\mathrm{pu})$


Sag or swell type

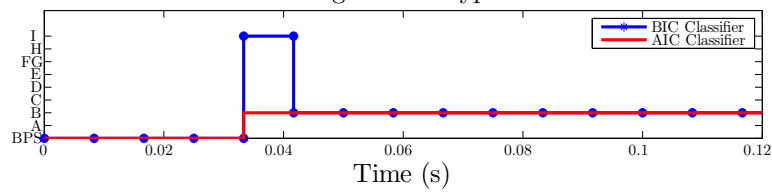

Fig. 12. Event 5 (2911): Voltage sag and swell classification. Comparison between ML classifiers using BIC and AIC penalty terms.

App classifiers, simulation results have shown that the ML classifier generally leads to the best classification performance. However, it should be mentioned that the approximate classifier can reach the performance of the ML classifier for particular cases, especially when the signal length $N$ is a multiple of a half-cycle. Concerning the penalty term, most simulations have shown that the classifier using the BIC penalty term outperforms the AIC-based classifier. Indeed, simulation results have shown that the AIC penalty term seems to overestimate the number of non-zero symmetrical components.

Regarding the computation complexity, the approximate classifier has a lower computation time than the ML one. Indeed, this latter classifier involves larger number of matrix multiplications in comparison to the ML classifier. Nevertheless, the performance of the approximate classifier critically depends on the number of samples. Specifically, the classifier performance rapidly degrades when the number of samples is not a multiple of a half-cycle.

Under quasi-stationary conditions, i.e. when the signal parameters are time-varying, the tracking of the signal class requires a small sample window length, $N$. Nevertheless, there is a natural tradeoff between the tracking and statistical performance. According to Monte Carlo simulation, for the approximate classifier, we advise to select a window length $N$ equal to a multiple of a half-cycle. In particular, to meet the the minimum sag and swell durations defined in the standard IEEE std. 1159, we recommend to choose a window length equal to a half-cycle. To easily meet this criterion, we also advise to choose a sampling frequency equal to $F_{s}=N f_{0}$ where $N>>2$ and $f_{0}$ is the signal frequency $\left(f_{0}=50 \mathrm{~Hz}\right.$ or $\left.f_{0}=60 \mathrm{~Hz}\right)$.

\section{CONCLUSION}

This paper dealt with power quality disturbances classification in three-phase unbalanced power systems with a particular focus on voltage sags and swells. A new classification approach based on Information Theoretical Criteria has been proposed. This approach is mainly based on two steps: 1) the signal pre-classification into one of 4 pre-classes, where each class contains one or various sags or swells types and depends on the number of non-zero symmetri- 
cal components, 2) the signature type classification using the ML estimate of the symmetrical components.

Regarding the proposed classifier, we have investigated the use of two Information Theoretical Criteria, namely the AIC and BIC. Simulation results have shown that the BIC criteria outperforms the AIC criteria in terms of average probability of correct classification for different data length and signal to noise ratio, whatever the classifier is. In particular, the analysis of the confusion matrices has indicated that the AIC seems to slightly overestimate the number of non-zero symmetrical component even for a large number of samples or signal to noise ratio. The results have also shown that the BIC classifier is robust against harmonic environment for moderate Total Harmonic Distortion (THD < 50\%).

For applications requiring a low computational complexity, we have also proposed an approximate classifier that involves fewer matrix multiplications and no matrix inversion. Simulations based on synthetic signals and real power systems data have clearly shown that, in most of the cases, the exact classifier exhibits better statistical performances than the approximate one. However, simulation results have also shown that the approximate classifier has a much lower computation complexity and can lead to near optimal performances when $N$ is a multiple of a half-cycle. Future investigations should deal with the evaluation of the proposed algorithms performances for the classification of other critical PQ disturbances.

\section{REFERENCES}

[1] J. Hu, J. Zhu, and G. Platt, "Smart grid the next generation electricity grid with power flow optimization and high power quality," in Proceedings of the 2011 IEEE ICEMS, Beijing (China), pp. 1-6, August 2011.

[2] H. Farhangi, "The path of the smart grid," IEEE Power and Energy Magazine, vol. 8, no. 1, pp. 18-28, February 2010.

[3] X. Fang, S. Misra, G. Xue, and D. Yang, "Smart grid the new and improved power grid: A survey," IEEE Communications Surveys \& Tutorials, vol. 14, no. 4, pp. 944-980, October 2012.

[4] I.-Y. Chung, D.-J. Won, J.-M. Kim, S.-J. Ahn, and S.-I. Moon, "Development of a network-based power quality diagnosis system," Electric Power Systems Research, vol. 77, pp. 1086-1094, June 2007.

[5] S. Chattopadhyay, M. Mitra, and S. Sengupta, Electric Power Quality. Springer, March 2011.

[6] H. Gharavi and R. Ghafurian, "Smart grid: The electric energy system of the future [scanning the issue]," Proceedings of the IEEE, vol. 99, $\mathrm{n}$. 6, pp. 917-921, June 2011.

[7] A. R. Bergen, Power Systems Analysis. Pearson Education India, July 2000.

[8] K. D. McBee and M. G. Simoes, "Utilizing a smart grid monitoring system to improve voltage quality of customers," IEEE Transactions on Smart Grid, vol. 3, no. 2, pp. 738-743, June 2012.

[9] IEEE, "IEEE approved draft guide for the interoperability of energy storage systems integrated with the electric power infrastructure," IEEE P2030.2/D9.0, pp. 1-136, June 2015.

[10] S. Amin and B. Wollenberg, "Toward a smart grid: Power delivery for the 21st century," IEEE Power and Energy Magazine, vol. 3, no. 5, pp. 34-41, September 2005.

[11] M. H. J. Bollen, I. Gu, S. Santoso, M. Mcgranaghan, P. Crossley, M. Ribeiro, and P. Ribeiro, "Bridging the gap between signal and power," IEEE Signal Processing Magazine, vol. 26, no. 4, pp. 12-31, July 2009.

[12] M. F. McGranaghan, D. R. Mueller, and M. J. Samotyj, "Voltage sags in industrial systems," IEEE Transactions on Industry Applications, vol. 29, no. 2, pp. 397-403, March-April 1993.

[13] "Ieee recommended practice for monitoring electric power quality," IEEE Std 1159-2009 (Revision of IEEE Std 1159-1995), pp. c1-81, June 2009.

[14] "Testing and measurement techniques-power quality measurement methods," International Electrotechnical Commission Standard 61000-4-30, January 2015.

[15] "Voltage characteristics of electricity supplied by public distribution systems,"

[16] M. Sun, S. Demirtas, and Z. Sahinoglu, "Joint voltage and phase unbalance detector for three phase power systems," IEEE Signal Processing Letters, vol. 20, no.1, pp. 11-14, January 2013.
[17] T. Routtenberg and L. Tong, "Networked detection of voltage imbalances for three-phase power systems," in Proceedings of the 2015 IEEE ISIE, Buzios-Rio de Janiero (Brazil), pp. 1345-1350, June 2015.

[18] M. H. J. Bollen, "Algorithms for characterizing measured three-phase unbalanced voltage dips," IEEE Transactions on Power Delivery, vol. 18, no. 3, pp. 937-944, July 2003.

[19] M. H. J. Bollen and E. Styvaktakis, "Characterization of three-phase unbalanced dips (as easy as one-two-three?)," in Proceedings of the 2000 IEEE IHQP, Orlando (USA), vol. 1, pp. 81-86, October 2000.

[20] L. Zhan and M. H. J. Bollen, "Characteristic of voltage dips (sags) in power systems," IEEE Transactions on Power Delivery, vol. 15, no. 2, pp. 827-832, April 2000.

[21] M. Madrigal and B. Rocha, "A contribution for characterizing measured three-phase unbalanced voltage sags algorithm," IEEE Transactions on Power Delivery, vol. 22, no. 3, pp. 1885-1890, July 2007.

[22] M. H. J. Bollen, Understanding Power Quality Problems, vol. 3. IEEE Press New York, October 1999.

[23] V. Ignatova, P. Granjon, and S. Bacha, "Space vector method for voltage dips and swells analysis," IEEE Transactions on Power Delivery, vol. 24, no. 4, pp. 2054-2061, October 2009.

[24] S. P. Valsan and K. S. Swarup, "High-speed fault classification in power lines: Theory and fpga-based implementation," IEEE Transactions on Industrial Electronics, vol. 56, pp. 1793-1800, May 2009.

[25] Z. Liu and Q. Zhang, "An approach to recognize the transient disturbances with spectral kurtosis," IEEE Transactions on Instrumentation and Measurement, vol. 63, no. 1, pp. 46-55, January 2014.

[26] M. D. Borrás, J. C. Bravo, and J. C. Montaño, "Disturbance ratio for optimal multi-event classification in power distribution networks," IEEE Transactions on Industrial Electronics, vol. 63, no. 5, pp. 3117-3124, January 2016.

[27] Z. Liu, Y. Cui, and W. Li, "A classification method for complex power quality disturbances using EEMD and rank wavelet SVM," IEEE Transactions on Smart Grid, vol. 6, no. 4, pp. 1678-1685, January 2015.

[28] A. Bíscaro, R. Pereira, M. Kezunovic, and J. Mantovani, "Integrated fault location and power-quality analysis in electric power distribution systems," IEEE Transactions on Power Delivery, vol. 31, no. 2, pp. 428436, March 2016.

[29] S. Khokhar, A. A. B. M. Zin, A. S. B. Mokhtar, and M. Pesaran, "A comprehensive overview on signal processing and artificial intelligence techniques applications in classification of power quality disturbances," Renewable and Sustainable Energy Reviews, vol. 51, pp. 1650-1663, November 2015.

[30] B. Biswal, M. Biswal, S. Mishra, and R. Jalaja, "Automatic classification of power quality events using balanced neural tree," IEEE Transactions on Industrial Electronics, vol. 61, pp. 521-530, January 2014.

[31] S. K. Meher and A. K. Pradhan, "Fuzzy classifiers for power quality events analysis," Electric Power Systems Research, vol. 80, pp. 71-76, September 2010.

[32] B. Biswal, P. K. Dash, and B. K. Panigrahi, "Power quality disturbance classification using fuzzy c-means algorithm and adaptive particle swarm optimization," IEEE Transactions on Industrial Electronics, vol. 56, pp. 212-220, January 2009.

[33] I. W. Lee and P. K. Dash, "S-transform-based intelligent system for classification of power quality disturbance signals," IEEE Transactions on Industrial Electronics, vol. 50, pp. 800-805, August 2003.

[34] C. Bhende, S. Mishra, and B. Panigrahi, "Detection and classification of power quality disturbances using s-transform and modular neural network," Electric Power Systems Research, vol. 78, pp. 122-128, December 2008.

[35] M. Valtierra-Rodriguez, R. de Jesus Romero-Troncoso, R. A. OsornioRios, and A. Garcia-Perez, "Detection and classification of single and combined power quality disturbances using neural networks," IEEE Transactions on Industrial Electronics, vol. 61, pp. 2473-2482, May 2014.

[36] M. Uyar, S. Yildirim, and M. T. Gencoglu, "An effective wavelet-based feature extraction method for classification of power quality disturbance signals," Electric Power Systems Research, vol. 78, pp. 1747-1755, October 2008.

[37] P. G. Axelberg, I. Y.-H. Gu, and M. H. J. Bollen, "Support vector machine for classification of voltage disturbances," IEEE Transactions on Power Delivery, vol. 22, pp. 1297-1303, July 2007.

[38] Z. Oubrahim, V. Choqueuse, Y. Amirat, and M. E. H. Benbouzid, "Classification of three-phase power disturbances based on model order selection in smart grid applications," in Proceedings of the 2016 IEEE IECON, Florence (Italy), pp. 5143-5148, December 2016. 
[39] IEEE, IEEE Standard Definitions for the Measurement of Electric Power Quantities Under Sinusoidal, Nonsinusoidal, Balanced, or Unbalanced Conditions. IEEE Press, March 2010.

[40] J. L. Blackburn, Symmetrical Components for Power Systems Engineering. CRC Press, June 1993.

[41] M. H. J. Bollen and L. Zhang, "Different methods for classification of three-phase unbalanced voltage dips due to faults," Electric Power Systems Research, vol. 66, no. 1, pp. 59-69, July 2003.

[42] A. G. Phadke and J. S. Thorp, Synchronized phasor measurements and their applications. Springer Science \& Business Media, 2008.

[43] P. Stoica, H. Li, and J. Li, "Amplitude estimation of sinusoidal signals: Survey, new results, and an application," IEEE Transactions on Signal Processing, vol. 48, pp. 338-352, February 2000.

[44] P. Stoica and R. L. Moses, Introduction to Spectral Analysis, vol. 1. Prentice hall Upper Saddle River, February 1997.

[45] G. H. Golub and V. Pereyra, "The differentiation of pseudo-inverses and nonlinear least squares problems whose variables separate," SIAM Journal on numerical analysis, vol. 10, pp. 413-432, April 1973.

[46] DOE/EPRI National Database Repository of Power System Events: Online; available at: http://pqmon.epri.com/disturbance_library/ (last accessed: May 2016).

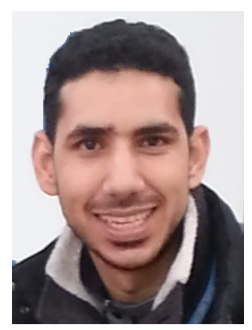

Zakarya Oubrahim was born in Errachidia Morocco, in 1987. He received the Engineering diploma degree in electrical engineering from the University of Technology of BelfortMontbéliard, Belfort, France, in 2013 and the M.Sc. degree in electrical engineering from the University of Lyon I, Lyon, France, in 2014. He is now a PhD degree candidate working on smart grids at the University of Brest, Brest, France.

His research interests include signal processing and statistics for power systems monitoring, smart grid solutions, measurement of power quality in multi-phase systems, control of renewable energy systems.



Vincent Choqueuse (S08-M09) was born in Brest, France, in 1981. He received the Dipl.Ing. and the M.Sc. degrees in 2004 and 2005, respectively, from the Troyes University of Technology, Troyes, France, and the $\mathrm{PhD}$ degree in 2008 from the University of Brest, Brest, France. Since September 2009, he has been an Associate Professor with the Institut Universitaire de Technologie of Brest, University of Brest, Brest, France, and a member of the Institut de Recherche Dupuy de Lôme - IRDL (FRE CNRS 3744).

Dr. Choqueuse research interests include signal processing and statistics for power systems monitoring, Smart-Grid, digital communication and digital audio.

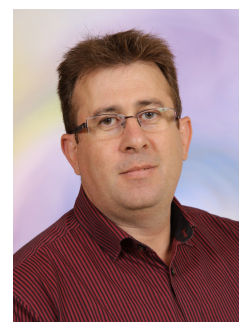

Yassine Amirat was born in Annaba, Algeria, in 1970. He received the B.Sc. and M.Sc. degrees in electrical engineering from the University of Annaba, Annaba, in 1994 and 1997, respectively. He was a lecturer at Annaba University from 2000 to 2010. He obtained the Ph.D. degree in wind turbine condition monitoring at the University of Brest, Brest, France in 2011.

$\mathrm{He}$ is currently an Associate Professor of Electrical Engineering at ISEN Brest (France). He is also an affiliated member at the Institut de Recherche Dupuy de Lôme IRDL (FRE CNRS 3744). His main research interests include electrical machines faults detection and diagnosis, fault tolerant control, and signal processing and statistics for power systems monitoring. $\mathrm{He}$ is also interested in renewable energy applications: wind turbines, marine current turbines and hybrid generation systems.

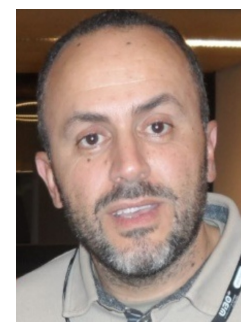

Mohamed El Hachemi Benbouzid (S92-M95SM98) was born in Batna, Algeria, in 1968. He received the $B$.Sc. degree in electrical engineering from the University of Batna, Batna, Algeria, in 1990, the M.Sc. and Ph.D. degrees in electrical and computer engineering from the $\mathrm{Na}$ tional Polytechnic Institute of Grenoble, Grenoble, France, in 1991 and 1994, respectively, and the Habilitation Diriger des Recherches degree from the University of Picardie Jules Verne, Amiens, France, in 2000.

After receiving the Ph.D. degree, he joined the Professional Institute of Amiens, University of Picardie Jules Verne, where he was an Associate Professor of electrical and computer engineering. Since September 2004, he has been with the Institut Universitaire de Technologie of Brest, University of Brest, Brest, France, where he is a Professor of electrical engineering. Prof. Benbouzid is also a Distinguished Professor at the Shanghai Maritime University, Shanghai, China. His main research interests and experience include analysis, design, and control of electric machines, variable-speed drives for traction, propulsion, and renewable energy applications, and fault diagnosis of electric machines.

Prof. Benbouzid is an IEEE Senior Member. He is the Editor-inChief of the International Journal on Energy Conversion (IRECON). $\mathrm{He}$ is also an Associate Editor of the IEEE Transactions on Energy Conversion, the IEEE Transactions on Industrial Electronics, the IEEE Transactions on Sustainable Energy, and the IEEE Transactions on Vehicular Technology. 